\title{
Area-wide management guidelines for Sterile Insect Technique developed through interdisciplinary modelling research
}

\author{
Hazel Parry ${ }^{a}$, Capon T. ${ }^{a}$, Clarke A. ${ }^{b}$, Crisp P. ${ }^{c}$, Hulthen A. ${ }^{a}$, Kandulu J. ${ }^{a}$, Kranz B. ${ }^{\text {, }}$ Loechel B. ${ }^{a}$,

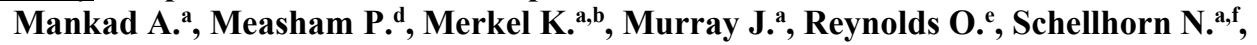 \\ Schwarzmueller F. ${ }^{a}$, Tam M. ${ }^{c}$, Tapsuwan S. ${ }^{a}$, van Klinken R. ${ }^{a}$, Whitten S. ${ }^{a}$ and Williams D. ${ }^{g}$ \\ ${ }^{a}$ Commonwealth Scientific and Industrial Research Organization, Australia \\ ${ }^{b}$ Queensland University of Technology, Australia \\ ${ }^{c}$ Primary Industries and Regions South Australia/The South Australian Research and Development Institute, \\ Australia \\ ${ }^{d}$ Hort Innovation, Australia \\ ${ }^{e}$ cesar, Australia \\ ${ }^{f}$ RapidAIM Pty Ltd, Australia \\ ${ }^{g}$ Agriculture Victoria, Australia
}

Email:.hazel.parry@,csiro.au

\begin{abstract}
New technologies for insect pest management, such as Sterile Insect Technique (SIT), present an opportunity to reduce chemical use in agricultural landscapes. However, they require socio-economic and ecological evaluation to ensure they are effective. We present a decision-making framework for sterile Queensland fruit fly 'Qfly' (Bactrocera tryoni) release informed by both economic and biophysical models as part of an area-wide management (AWM) program with SIT. The conceptual framework presented here guided the development of economic and biophysical models to meet the objective of informing management strategies.
\end{abstract}

Our economic modelling has provided an important assessment of the feasibility and opportunities for AWM and SIT at the scale of three case study regions in southern Australia (Sunraysia, Murray/Goulburn valley (MGV) and Riverland). We found that there are three potential economically-viable implementation strategies for AWM of Qfly including SIT: outbreak eradication (and potentially maintenance of area freedom, should legislation allow); direct substitution for existing management techniques in spatiallyisolated contexts (including in support of industry-specific market access); and more effective management in an urban context than existing ad hoc approaches.

In terms of releases of sterile males, our biophysical research has generated valuable risk maps for three case study regions. An understanding of the spatial and temporal risk of Qfly occurrence for a given target region is essential to the development of a future tool or system for planning sterile fly releases, as well as for ongoing management and monitoring programs. As a minimum, knowledge of the land use, climatic suitability and host phenology in a region is required. Ideally, this should incorporate the construction of risk maps that can inform decision-making for releases. The spatial simulation modelling showed that complex landscapes, containing a higher diversity of fruiting hosts across different seasons, will pose greater challenges in achieving population suppression and adoption of AWM strategies in readiness for SIT. Urban areas pose a significant challenge, as these areas provide a reservoir of flies throughout the year with potential to move into nearby crops. Therefore, it is important that they are managed as part of an AWM approach (potentially employing SIT) in order to effectively suppress flies across a landscape. The model identifies potential hotspots and bottlenecks in space and time which can be used to develop more targeted and effective SIT release strategies. We show that an urban treatment is most effective when reducing populations in late winter/early spring before they can move into the agricultural area.

Our work highlights the importance of a conceptual framework that provides a broad consideration of the economic, social, and biological feasibility of a SIT program prior to the release of sterile flies using modelling approaches, as well as an ongoing need to consider the socioeconomic and biophysical components of the system for sterile Qfly releases to be successful. This work led to the development of online guidelines for area-wide management (AWM) of Qfly incorporating Sterile Insect Technique (SIT) in Australia www.area-wide-management.com.au.

Keywords: Bioeconomic modelling, Spatial simulation, Queensland fruit fly, Bactrocera tryoni 
Parry, H.R. et al., Producing area-wide management guidelines for Sterile Insect Technique with interdisciplinary modelling research

\section{INTRODUCTION}

Established by Hort Innovation and the Government of South Australia, SITplus aims to deliver and implement sustainable cost-effective solutions to Queensland Fruit fly (Qfly) management across Australia and New Zealand through an integrated area-wide approach based on the deployment of Sterile Insect Technique (SIT) and enhanced ecological understanding. The 'plus' provides the framework of integrated, ecological/behavioural support science to ensure maximum effectiveness of SIT. This framework incorporates socio-economic as well as biophysical understanding to develop strategic considerations for releases. Integrating knowledge from different disciplines is a major challenge, but is required to fully inform the management of Qfly using AWM and SIT.

Modelling is important to both an up-front consideration of the feasibility of a SIT program in a target area, and also to three of the major components of the strategic considerations for releases: socio-economic context, landscape context and fly biology and ecology. These factors need to be considered to: a) assess the applicability of SIT to a given situation; and b) enable the SIT program to achieve its objectives across a target area. For example, in a given scenario modelling can help define the scale, extent and frequency of releases that will be required, assess the economic value proposition of deploying SIT, and determine the implications for efficacy of existing Qfly management activities alongside SIT.

\section{METHODS}

In order to consider the steps needed to produce and deploy sterile Qfly males and how this decision-making is best undertaken, we constructed a conceptual model of the decision-making framework for SIT releases (Figure 1). This shows where modelling, both biophysical and economic, fits in relation to the overall operation of producing and deploying sterile males for managing Qfly, and the importance of the model outputs to strategic considerations for releases.

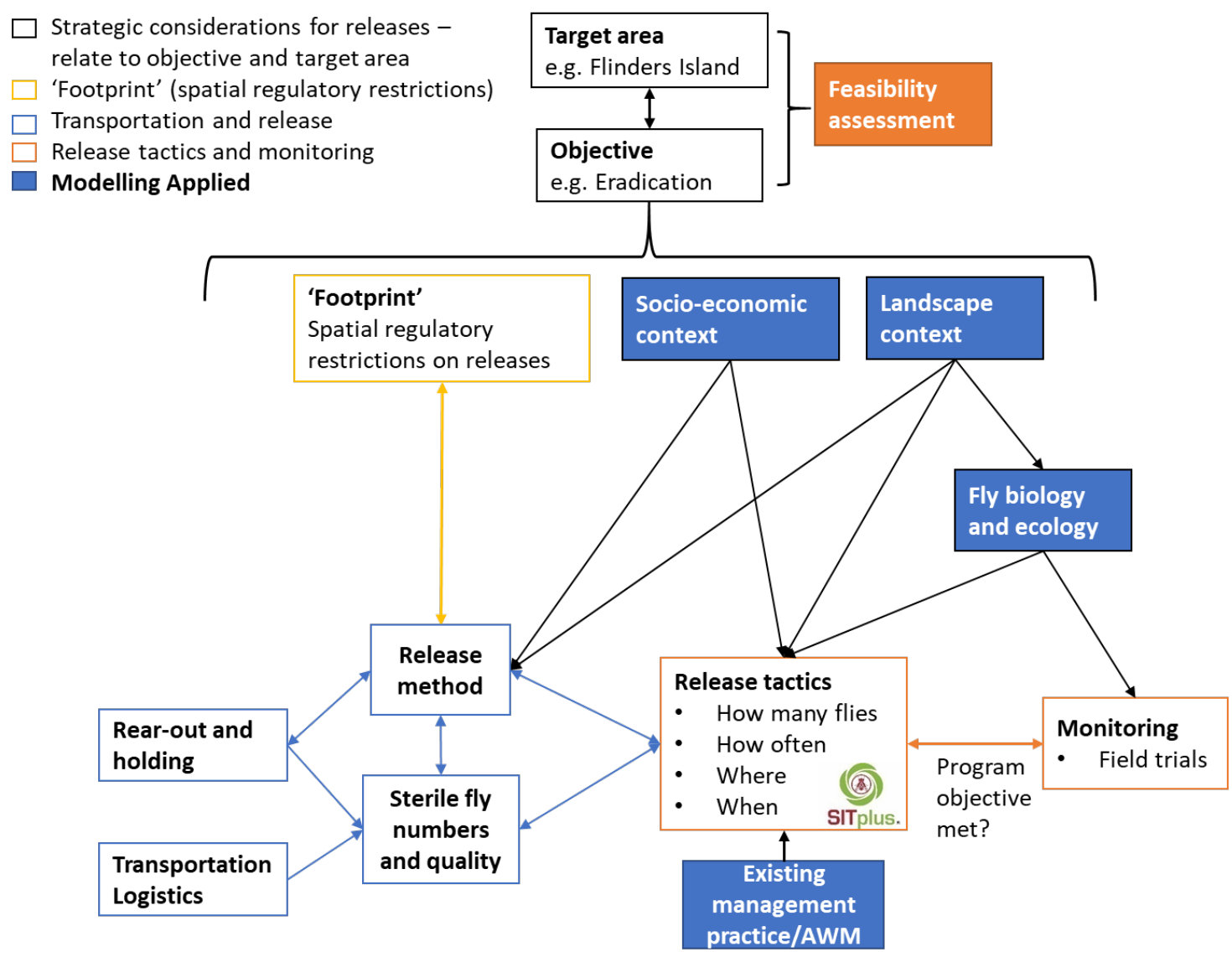

Figure 1. The complexity of decision-making for sterile male Queensland fruit fly releases, showing both the need for strategic as well as tactical decision making. 
Parry, H.R. et al., Producing area-wide management guidelines for Sterile Insect Technique with interdisciplinary modelling research

This framework highlights the key socio-economic, landscape and biophysical considerations in any given scenario that need to be systematically evaluated, as well as factors to consider in evaluating the feasibility of management objectives in a target area. This can be achieved by economic and biophysical modelling approaches as shown, alongside social science studies. These considerations should be adopted as part of a strategic planning process to produce and deploy sterile males for managing Qfly. We applied modelling within this framework to three case study regions in southern Australia (Sunraysia, Murray/Goulburn valley (MGV) and Riverland), which have experienced Qfly invasion in recent years (Riverland is still currently fruit fly free). Our economic modelling considered the feasibility of AWM and SIT as well as willingness to pay for implementation by both growers and local communities. Our biophysical modelling used habitat suitability modelling with expert elicitation and Bayesian belief networks to identify areas that are most at risk from Qfly throughout the year. A biophysical simulation model of Qfly population dynamics and dispersal was then developed to consider in which landscapes particular management strategies were likely to be more or less effective.

\section{RESULTS}

\subsection{Outbreak eradication}

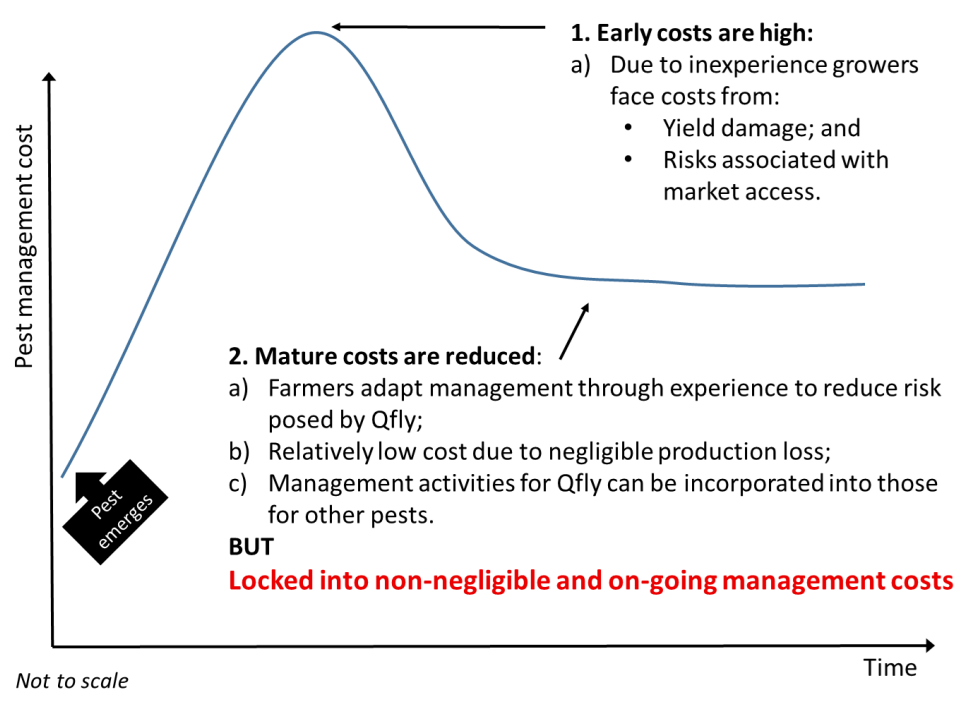

Figure 2. Queensland fruit fly management costs to growers over time once it invades and becomes established.

introducing AWM at that point requires a higher level of management adoption with commensurate costs (Figure 3).

Growers and regional groups must manage the pest for some time beyond the introduction of an AWM program, to suppress fly populations to support SIT implementation, and beyond SIT for at least some period to avoid the potential for costly future damages or risk to domestic and export market access (the length of which will depend on whether suppression or eradication is the goal). Furthermore, the baseline ongoing costs of managing for
Our economic modelling (Tam et al., submitted) indicated that investment in outbreak eradication (inclusive of SIT) delivers a strong positive economic payoff and thus is a suitable objective for SIT and AWM. For designated Pest Free Areas (PFAs), such as the Riverland in South Australia, preventing Qfly establishment averts both the initial high cost of a Qfly invasion on production as well as the ongoing costs of management,

while maintaining the benefits from PFA status (Figure 2).

The future trajectory of management becomes locked in once Qfly are established. No matter the objective,

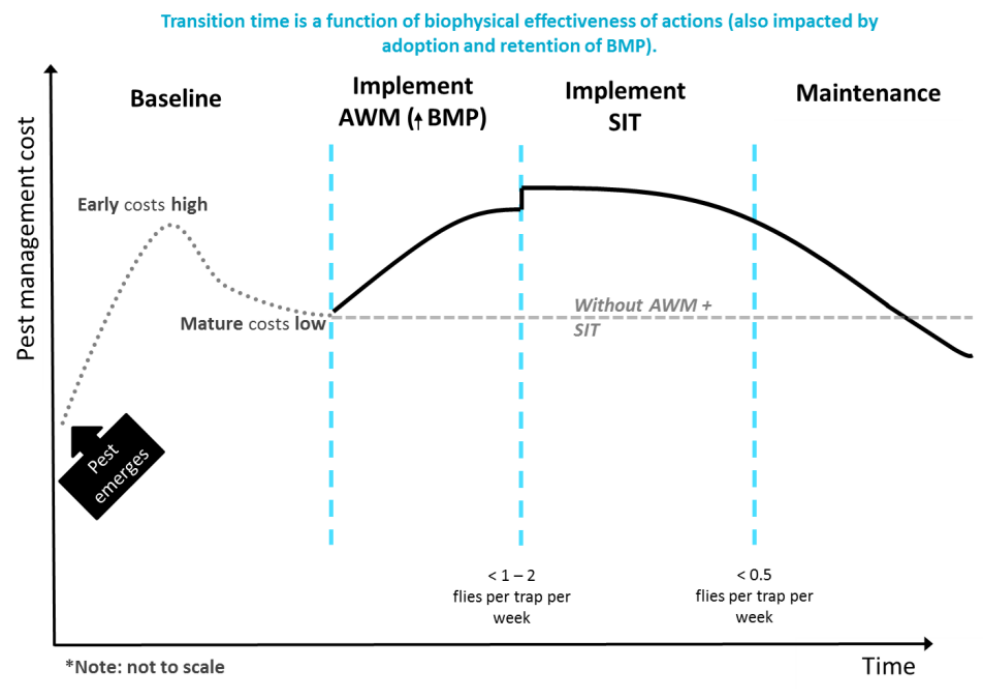

Figure 3. Queensland fruit fly management costs to growers over time once it invades and becomes established, including AWM and SIT. 
Parry, H.R. et al., Producing area-wide management guidelines for Sterile Insect Technique with interdisciplinary modelling research

Qfly are relatively low (although non-negligible, Figure 2), but with negligible additional ongoing production losses once growers incorporate Qfly management into their wider pest management regime on-farm. We estimate that introducing AWM and eventually SIT incurs additional early costs, while delivering uncertain and relatively small future economic benefits in areas where Qfly have become established (Figure 3).

\subsection{Direct substitution for existing management in spatially-isolated areas}

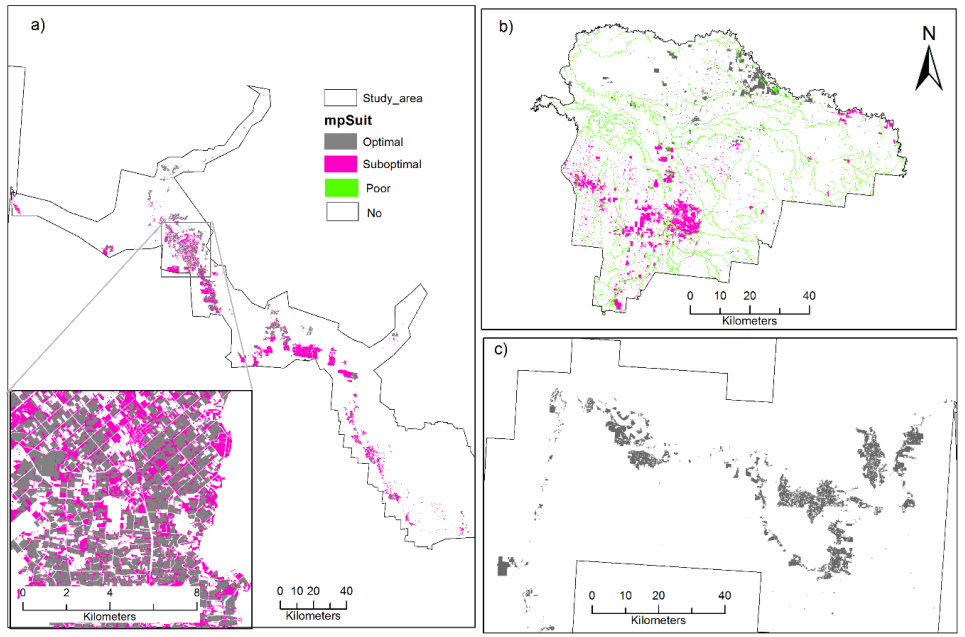

Figure 4. Habitat suitability maps for a) Sunraysia (with insert showing an area in finer detail), b) MGV, and c) Riverland regions. 'mpSuit' shows the level of suitability of habitat across the region on an annual basis, as estimated by the model.
Direct substitution for some existing management practices where SIT provides a more cost-effective option (including in support of industry-specific market access) is a second feasible objective for SIT, and could potentially reduce initial and/or maintenance costs following pest outbreak and establishment. This is only likely to be applicable in limited situations (spatiallyisolated areas, from a Qfly perspective) and where SIT is effective enough to enable other costly activities to be reduced/stopped.

Risk maps (Figure 4) show areas of high likelihood of Qfly persistence based on expert knowledge of climatic suitability, environment and

management and the effects those factors have on Qfly populations in general across an annual timeframe (Murray et al. submitted). These risk maps, which consider a range of climatic and habitat factors, are useful in helping decide where AWM using SIT is most practicable and how SIT might best be implemented there. The habitat suitability model was primarily driven by host availability/quality. A secondary driver was climatic stress. The susceptibility model gives a month-by-month estimate of how much risk is posed to particular commodities, depending on the time of year, other commodities within the vicinity (and thus potential movement of the flies targeting other hosts) and broad scenarios of management effectiveness. In these maps, Qfly trapping data was integrated to represent source pressure throughout the year.
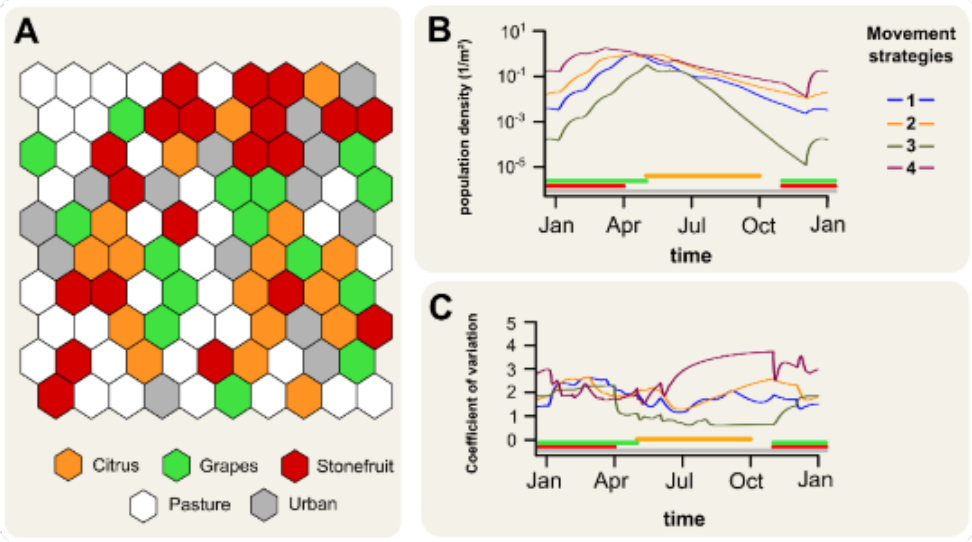

Figure 5. Example of one of the landscapes used in the spatial simulation model (A), the respective population dynamics (in flies per $\mathrm{m}^{2}$ ) under different movement scenarios (B) and the between patch variation in population densities expressed as Coefficient of Variation (C). 
Parry, H.R. et al., Producing area-wide management guidelines for Sterile Insect Technique with interdisciplinary modelling research

\subsection{Effective management in an urban context}

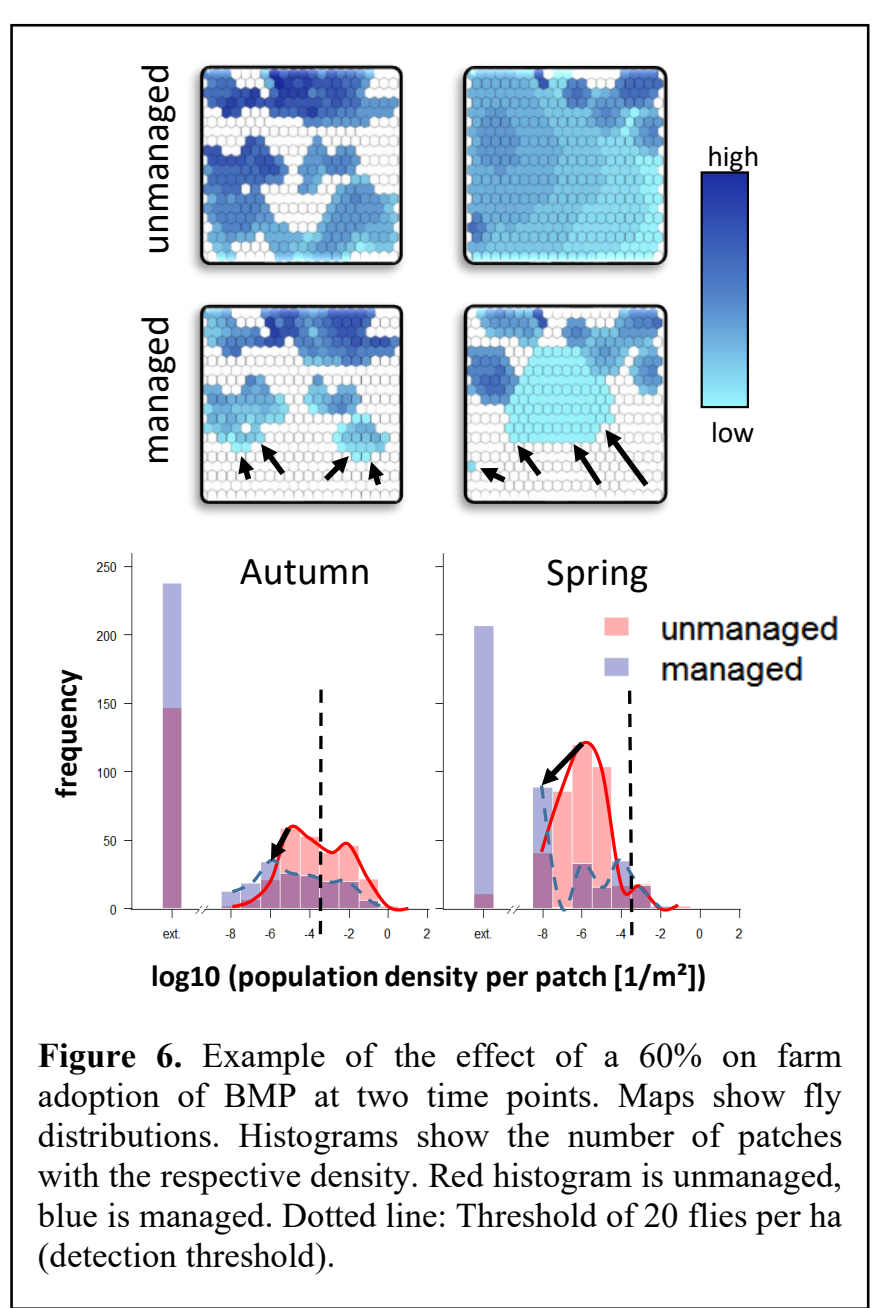

A spatial simulation model helped identify key factors that contribute to larger and more sustained populations in different landscape contexts (Figure 5). In terms of population dynamics, the key findings from the model indicated that the seasonal availability of fruit is the main driver of Qfly population size and persistence, with more continuous (and high quality) resources leading to larger, more persistent, populations (Schwarzmueller et al., in press). Moreover, continuous but low density resources such as fruit trees in urban areas can also be important; this was supported by comparison with an analysis of Ag Vic and NSW DPI trapping data and data from our own field observations: even if a host has only a small amount of fruit available it can still be a significant source of Qfly populations.

The spatial simulation model also compared management strategies and their pestsuppression effectiveness in different contexts (Figure 6). The model showed that higher levels of adoption of best management practice (BMP) on farm are more beneficial to suppress pests, but in complex landscapes in particular the level of adoption needed to achieve success needs to be relatively high (Schwarzmueller et al., submitted). In such landscapes, involving urban residents/backyard growers into an AWM program is crucial for pest suppression as such levels of adoption of BMP by commercial growers may be unattainable. The modelling also showed that an urban treatment is most effective when reducing populations in late winter/early spring before they can move into the agricultural area.

\section{DISCUSSION AND CONCLUSIONS}

This paper describes a conceptual framework designed to assess the effective deployment of AWM and SIT using modelling approaches. This approach is vital to employ in order to enable these non-chemical forms of pest management to be a successful and viable alternative method of Qfly control.

Currently, while Qfly suppression may be achieved through AWM effort as demonstrated by our biophysical models, management of established populations across large areas to achieve eradication and subsequent improved market access based on pest free area status is unlikely to be an appropriate objective. This is because there is unlikely to be sufficient positive economic return from market-access or landholder cost savings to compensate for the additional management costs of the incorporation of SIT.

The framework and the models that support it indicate that it is important to evaluate the applicability and benefits of SIT in a given scenario, and to set clear and measurable objectives across the target area that can inform the deployment strategy (as well as a means to evaluate success). To this end, a set of guidelines were developed and produced, based on the outputs of the research presented here (www.area-widemanagement.com.au) which have successfully provided a tool by which communities can ensure they are in the best position to consider employing SIT, as part of an AWM strategy for Qfly. Such a communication device is necessary to distill complex research findings, particularly from models, into information that can provide useful guidance for actions. 
Parry, H.R. et al., Producing area-wide management guidelines for Sterile Insect Technique with interdisciplinary modelling research

\section{ACKNOWLEDGMENTS}

The Adaptive Area wide management of Qfly using SIT was delivered by Hort Innovation in partnership with CSIRO, and was supported by funding from the Australian Government Department of Agriculture as part of its Rural R\&D for Profit program (14-01-047). Further partners included QUT, Agriculture Victoria, NSW DPI, PIRSA, SARDI, Wine Australia and BioFly.

\section{REFERENCES}

Murray, J., van Klinken, R., and Clarke, A. (submitted) Developing spatially explicit risk maps for Bactrocera tryoni (Diptera: Tephritidae) in southern Australia to facilitate area-wide management. Agriculture Ecosystems and Environment

Schwarzmueller, F., Schellhorn, N.A., and Parry, H.R. (in press). Resource landscapes and movement strategy shape Queensland Fruit Fly population dynamics. Landscape Ecology.

Schwarzmueller, F., Reynolds, O., Schellhorn, N.A., and Parry, H.R. (submitted). Area-wide management of Queensland Fruit Fly: How, where and when to manage. Ecological Applications.

Tam, M.K., Capon, T., Whitten, S., Tapsuwan, S., Kandulu, J.. and Meansham, P. (submitted). Assessing the economic benefit of Area Wide Management and the Sterile Insect Technique for the Queensland fruit fly in pest-free vs. endemic regions of south-east Australia. AJARE. 